\title{
成年耳细胞克隆山羊(Capra hircus)*
}

\author{
郭继䑣 ${ }^{(1 * *}$ 安志兴 ${ }^{(2)}$ 李 显 ${ }^{(1)}$ 李雪峰 ${ }^{(2)}$ 李裕强 ${ }^{(2)}$ \\ 郭泽坤 (2) 张 涌 $^{(2)}$
}

(1)内蒙古大学生命科学学院, 呼和浩特 010021; (2)西北农林科技大学生物工程研究所, 杨陵 712100)

\begin{abstract}
摘要繁殖季节采集关中奶山羊卵巢, 采集获得可用卵母细胞 5.5 枚/卯巢(1815/330). 经约 $20 \mathrm{~h}$ 成熟培养, 第一极体排放率 66.17\%(1201/1815). 将有类第二极体排出结构的 成熟卵母细胞去核, 去核率 75.44\%(906/1201). 培养济宁青山羊耳部皮肤成纤维细胞传 2 代后液氮冷冻, 解冻培养 $3 \sim 6$ 代, 用 $0.5 \%$ FBS 饥饿 $2 \sim 10 \mathrm{~d}$ 作为供体细胞. 利用卵母 细胞胞质内注射法将分离的供体细胞核胞体注入到去核卵母细胞内, 注核成功率 98.12\%(889/906). 克隆胚胎用 $5 \mu \mathrm{mol} / \mathrm{L}$ 离子酶素激活 $4.5 \mathrm{~min}$, 在含 $2 \mathrm{mmol} / \mathrm{L} \mathrm{6}$-二甲氨 基嘌呤 (6 dimethylaminopurine, 6-DMAP) 的培养液中培养 $3 \mathrm{~h}$, 然后在 mCR $1 \mathrm{aaBF}$ 培养液 中培养 $36 \mathrm{~h}$, 卵裂卒 76.69\%(645/841). 其中由未经冷藏处理供体细胞克隆得到 308 枚胚 胎, 激活后卵裂率、4-细胞发育率、囊胚发育率分别为 68.5\%(211/308), 59.72\% (126/211)和 $17.46 \%$ (22/126); 另外, 由 $4^{\circ} \mathrm{C}$ 冷藏处理 $24 \mathrm{~h}$ 供体细胞获得的 109 枚克隆胚 胎, 激活率、4-细胞率、囊胚率分别为 72.48\%(79/109), 53.16\%(42/79)和 $19.05 \%(8 / 42)$. 统 计学分析表明, $4^{\circ} \mathrm{C}$ 处理供体细胞对克隆胚胎的早期发育没有显著影响. 将 102 枚发育 至 4-细胞期的克隆肧胎移植到 17 只自然发情 2 3 d 的受体山羊输卵管, 其中非冷藏供 体细胞克隆的 84 枚胚胎移植后没有产仔. 18 枚冷藏体细胞克隆的胚胎移植获得 1 只发 育足月的克隆山羊. 将冷藏或非冷藏处理供体细胞克隆得到的 19 枚体外发育囊胚移植 到自然发情 6 $8 \mathrm{~d}$ 的受体山羊, 结果无一产仔. 未经冷藏处理供体细胞克隆得到的 18 枚体外发育桑椹肧移植到自然发情 $5 \mathrm{~d}$ 受体山羊后获得 1 只足月羔羊. 微卫星引物 PCR 扩增结果证实 2 只克隆山羊来源于同一供体细胞.
\end{abstract}

\section{关键词 山羊 耳皮肤成纤维细胞 细胞质内直接注射 核移植 克隆山羊}

自 1997 年, 英国科学家 Wilmut 等人 ${ }^{[1]}$ 首次报道成功地获得了成年体细胞克隆绵羊 Dolly 后, 体细胞克隆动物的研究进展迅速. 在美国, Wakayama 等人 ${ }^{[2]}$ 报道用细胞质内直接注射法, 获得了卵丘细胞克隆小鼠 30 余只. 实验同时还用睪丸支持细胞和脑神经细胞进行克隆, 但只 获得了克隆胎儿. 同年, 日本科学家用培养的卵丘细胞和输卵管上皮细胞进行克隆, 结果获得 了两种细胞克隆牛 8 只 ${ }^{[3]}$. 此后有研究报道, 用一头约 13 岁牛的颗粒细胞传 $4 \sim 8$ 代后克隆得 到两头牛犊 ${ }^{[4]}$. 牛乳腺上皮原代培养细胞和耳部皮肤成纤维细胞 ${ }^{[5]}$ 以及培养的肌肉细胞 ${ }^{[6]}$ 进 行克隆也都获得了成功. 为了转基因体细胞克隆的商业化, 胎儿体细胞克隆研究也在进行. 在

2001-06-08 收稿, 2001-07-20 收修改稿

*国家自然科学基金(批准号: 39830280)重点资助项目和“863”高技术资助项目

** E-mail: gjitong@hotmail.com 
获得胎儿成纤维细胞克隆绵羊后 ${ }^{[7]}$, 相继在牛 ${ }^{[8 \sim 10]}$ 、山羊 ${ }^{[11]}$ 和猪 ${ }^{[12]}$ 也获得成功. 在这些研究中, 采用的体细胞大多是新鲜或经短期培养传代后的细胞. 后来报道用一头 17 岁牛的耳部皮肤成 纤维细胞培养达 3 个月传 15 代后, 经核移植也得到了体细胞克隆牛 ${ }^{[13]}$. 这些研究表明, 分化 的体细胞甚至是分化末端的体细胞仍具有全能性, 在卵母细胞胞质的作用下可以重新发育形 成一个新个体.

体细胞克隆山羊的研究报道很少, 成年耳皮肤体细胞克隆山羊成功的报道尚未见到. 有 报道用体内 ${ }^{[11,14]}$ 和体外 ${ }^{[15]}$ 成熟的山羊卵母细胞作核移植受体获得胎儿体细胞克隆山羊. 这些 研究采用的核移植方法是常规的电融合，电融合核移植的效率目前还较低 ${ }^{[13,14]}$.

本研究用传代培养冷冻后的成年山羊耳皮肤成纤维细胞作核移植供体细胞, 比较了冷藏 处理供体细胞对克隆胚发育的影响, 并研究了用细胞质内直接注射法进行体细胞克隆山羊的 可行性.

\section{1 材料与方法}

\section{1 屠宰山羊卵巢的采集、卵母细胞体外成熟 ${ }^{[16]}$ 和去核}

从陕西三原采集繁殖季节 $\left(10\right.$ 月至翌年 1 月)屠宰的山羊卵巢，置于 $20^{\circ} \mathrm{C} \sim 30^{\circ} \mathrm{C}$ 含 0.32 $\mathrm{mg} / \mathrm{mL}$ 硫酸庆大霉素的生理盐水中, 在 6 8 h 内运回实验室. 用剃须刀片切开卵巢表面 1 5 $\mathrm{mm}$ 大小的卵泡, 收集山羊卵巢卵丘-卵母细胞复合体 $(\mathrm{COCs})$. 在 $38.5^{\circ} \mathrm{C}, 5 \% \mathrm{CO}_{2}$ 的空气和饱和

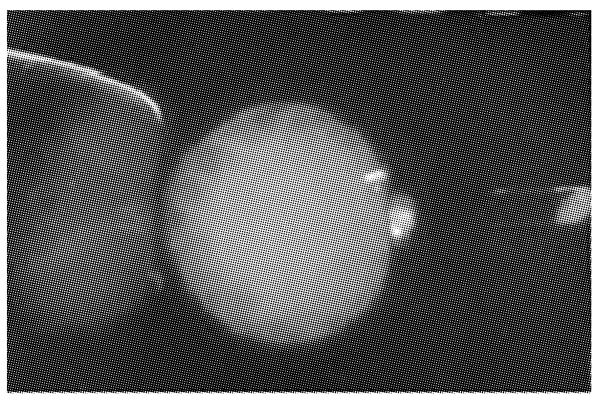

图 1 成熟卵母细胞去核前 Hoechst33342 染 色，示突出的类第二极体结构

湿度条件下培养 $20 \mathrm{~h}$, 体外成熟. 成熟液 TCM 199(Gibco, MM199)添加 10 mmol/L Hepes (Sigma), $0.38 \mathrm{mmol} / \mathrm{L}$ 丙酮酸钠 $(\mathrm{Sigma}), 50 \mu \mathrm{g} / \mathrm{mL}$ 硫酸庆大 霉素(Sigma), 10\%FBS (HyClone), $1 \mu \mathrm{g} / \mathrm{mL} 17-\beta \mathrm{E}_{2}$ (Sigma)和 $0.075 \mathrm{IU}$ FSH\&LH/mL (HMG, Serono), $10 \mathrm{ng} / \mathrm{mL}$ EGF(GIBCO).

成熟培养 $20 \mathrm{~h}$ 左右, 选择排放第一极体的卵 母细胞, 在 $5 \mu \mathrm{g} / \mathrm{mL}$ 细胞松弛素 B(Sigma)的培养液 中去核. 在显微镜下抽吸第一极体及其附近细胞 质表面突出的泡状结构即类第二极体排出结构(图 1)，去核后的卵母细胞置于培养箱中恢复培养.

\section{2 供体细胞的准备 ${ }^{[17]}$}

用无菌方法采集一小块济宁青山羊耳部皮肤，经过 $0.05 \%$ 过氧乙酸浸泡 $30 \mathrm{~s}$ 杀菌后在 PBS 中剪碎. 碎块接种于 $\mathrm{DMEM}(\mathrm{GIBCO}$, 添加 $10 \% \mathrm{FBS})$ 培养液中, $38.5^{\circ} \mathrm{C}, 5 \% \mathrm{CO}_{2}$ 的空气, 饱和湿 度下培养. 当培养细胞生长铺满培养瓶底面时, 用含 $0.25 \%$ 胰蛋白酶(GIBCO)和 $0.02 \% \mathrm{EDTA}$ (Sigma)的 $\mathrm{HBSS}$ (无 $\mathrm{Ca}^{2+}, \mathrm{Mg}^{2+}$, Sigma)消化培养细胞, 用吸管吹打使其快速悬浮. 离心悬液后 用培养液洗涤细胞沉淀. 以(1 5) $\times 10^{6}$ 个细胞/瓶重悬并接种于 $25 \mathrm{~mL}$ 培养瓶中传代培养, 如此 可以多次传代. 传代细胞以 $10^{6} \sim 10^{7}$ 个细胞 $/ \mathrm{mL}$ 悬浮在含 $10 \%$ 二甲基亚砜(DMSO, Sigma)的培 养液(含 $10 \% \mathrm{FBS}$ )中, $-20^{\circ} \mathrm{C}$ 放置 1 2 h, 结冰后在液氮表面停滞 $15 \mathrm{~min}$, 迅速投入液氮长期保存 ${ }^{[18]}$. 核移植前解冻接种在 24 孔培养板培养. 皮肤成纤维细胞传 2 代后冷冻, 然后解冻培养到 3 6 代用于核移植. 用前在含 $0.5 \%$ FBS 的 DMEM 培养液中培养 2 10 d. 
核移植前用胰蛋白酶消化, 悬于 $10 \% \operatorname{PVP}\left(360000\right.$, Sigma)注核液 ${ }^{[19]}$ 中, 部分细胞直接用 于核移植，部分细胞置于 $4^{\circ} \mathrm{C} 24 \mathrm{~h}$ 后用于核移植.

\section{3 核移植和克隆胚胎的激活与体外培养}

显微镜下, 用注核针反复抽吸供体细胞使其破膜成核胞体. 在添加 $15 \mathrm{mmol} / \mathrm{L} \mathrm{Hepes}$ (Sigma) 的 TCM 199(GIBCO, HM199)培养液中将 1 个核胞体 注人到 1 个去核卵母细胞胞质内(图 2), 一批可完成注 核 20 枚. 注核后立即洗涤克隆胚胎并放回培养箱培 养.

将注核胚胎恢复培养 3 6 h, 用 $5 \mu \mathrm{mol} / \mathrm{L}$ 离子酶 素 (Sigma) 激活 $4.5 \mathrm{~min}$, 迅速放人含 $2 \mathrm{mmol} / \mathrm{L}$ 6-DMAP(Sigma)(I-6DMAP)的培养液中培养 $3 \mathrm{~h}$. 培养 液为 $\mathrm{mCR} 1 \mathrm{aaBF}$, 即 $\mathrm{CR} 1 \mathrm{aa}^{[20]}$ 中添加 $1.19 \mathrm{mmol} / \mathrm{L}$ $\mathrm{KH}_{2} \mathrm{PO}_{4}, 1.5 \mathrm{mmol} / \mathrm{L}$ D-Glucose, $0.80 \mathrm{mmol} / \mathrm{L} \mathrm{MgCl}_{2}$,

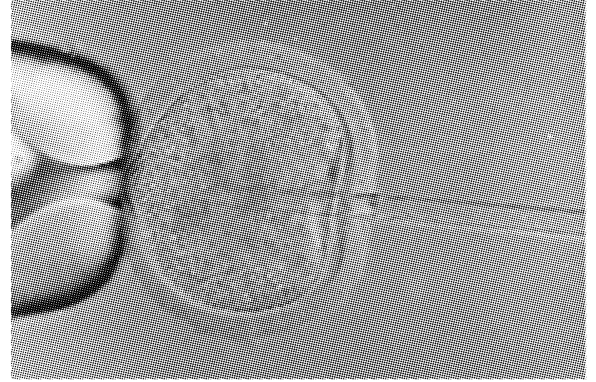

图 2 卵母细胞胞质内直接注射

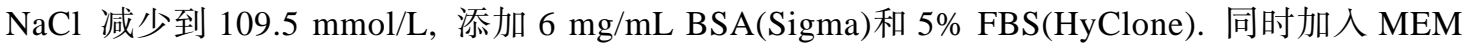
氨基酸溶液 (GIBCO)和 MEM 非必需氨基酸溶液(GIBCO). 激活后的胚胎用培养液洗涤, 在 $38.5^{\circ} \mathrm{C}, 5 \% \mathrm{CO}_{2}$ 的空气、饱和湿度下培养 $36 \mathrm{~h}$ 检查卵裂率. 卵裂的胚胎移人卵丘细胞单层共培 养系统中进一步培养, 每隔 $48 \mathrm{~h}$ 半量换液 1 次. 培养 $9 \mathrm{~d}$ 时检查囊肧发育率.

\section{4 克隆胚胎的外科手术移植}

选择形态发育好的 4-细胞克隆胚胎, 通过外科手术移植到自然发情 2 3 d 受体山羊输卵管 伞部, 每侧移植 3 枚. 发育的桑椹胚或囊胚用同样方法移植到自然发情 $5 \mathrm{~d}$ 或 $6 \sim 8 \mathrm{~d}$ 的受体山 羊子宫角. 桑椹胚也为双侧移植, 每侧移植 3 枚. 囊胚移植到黄体发育好的一侧.

\section{5 克隆山羊个体的微卫星 DNA 检测分析}

用山羊微卫星引物 ${ }^{[21 ~ 23]}$ (上海博亚生物技术有限公司合成)SR-CRSP1, SR-CRSP5, SRCRSP6, SR-CRSP7, SR-CRSP24进行核供体细胞、克隆山羊、克隆山羊受体和对照山羊总 DNA PCR 扩增. 扩增产物在 $6 \%$ 的变性聚丙烯酰胺测序胶电泳, $\mathrm{AgNO}_{3}$ 染色后显色 ${ }^{[24]}$ 检测扩增结果.

\section{2 结果}

\section{1 山羊卵母细胞胞质内直接注射法进行体细胞克隆的效率}

研究用繁殖季节采集的 330 个不知遗传背景和生殖状态的关中奶山羊卵巢, 采集得到可用于 成熟培养的卵母细胞 1815 枚, 平均采卵 5.5 枚/卵巢(1815/330). 约经 $20 \mathrm{~h}$ 成熟培养后, 机械吹吸 去除颗粒细胞, 统计有第一极体排放的卵母细胞 1201 枚, 体外成熟率 66.17\%(1201/ 1815)(表 1). 山羊卵母细胞经体外室温处理后, 多有类第二极体排出的结构, 成熟卵母细胞的中期染色体就位 于其中(图 1). 将具有此结构的卵母细胞去核，成功去核 906 枚，去核率 75.44\%(906/1201). 去核卵 母细胞在体外培养恢复后开始注核, 成功注核 889 枚, 注核成功率 98.12\%(889/906). 将注核成功 的卵母细胞在体外培养 3 6 h 后激活, 激活后存活的克隆胚胎 841 枚体外培养 $36 \mathrm{~h}$, 卵裂 645 枚, 卵裂率76.69\%(645/841). 结果表明, 采集的屠宰山羊卵巢在经过 6 8 h 运输后, 其中的卵母细胞不 仅可用于体外成熟, 而且成熟后的卵母细胞同样可用作体细胞克隆受体细胞. 结果进一步表明, 卵母细胞胞质内直接注射法可以高效重建体细胞克隆胚胎. 
表 1 体外成熟山羊卵母细胞胞质内注射核移植效率

\begin{tabular}{|c|c|c|c|}
\hline 采集卵巢数 & 330 & & \\
\hline 收集卵母细胞数 & 1815 & 平均采集 & 5.5 枚/卵巢 \\
\hline 体外成熟卵母细胞数 & 1201 & 体外成熟率 & $66.17 \%$ \\
\hline 去核卵母细胞数 & 906 & 去核率 & $75.44 \%$ \\
\hline 成功注核数 & 889 & 注核率 & $98.12 \%$ \\
\hline 激活的克隆胚胎数 & 841 & & \\
\hline 卵裂胚胎数 & 645 & 卵裂率 & $76.69 \%$ \\
\hline
\end{tabular}

\section{2 新鲜和冷藏的耳皮肤成纤维细胞克隆胚胎的发育结果比较}

用成年山羊耳部皮肤培养的成纤维细胞作供体，核移植前将消化分散的细胞分为两组， 一组直接用于核移植, 另一组在 $4^{\circ} \mathrm{C}$ 放置 $24 \mathrm{~h}$ 后用于核移植. 结果没有经过冷藏的细胞克隆重 建胚胎 308 枚，激活后的卵裂率是 68.5\%(211/308)，4-细胞的发育率是 59.72\%(126/211)，囊胚 发育率 $17.46 \%(22 / 126)$, 而经过 $4^{\circ} \mathrm{C}$ 冷藏的体细胞克隆得到胚胎 109 枚, 相应的激活率、4-细 胞率、囊胚率分别为 72.48\%(79/109), 53.16\%(42/79)和 19.05\%(8/42)(表 2). 统计学分析表明, 在 室温放置和 $4^{\circ} \mathrm{C}$ 放置的细胞对克隆后胚胎的早期发育没有显著影响. 但观察发现, 冷藏后的体细 胞克隆胚胎 4-细胞的发育形态比未处理的要好许多(图 3).

表 2 不同处理对山羊耳皮肤成纤维细胞克隆胚胎早期发育的影响 ${ }^{\text {a) }}$

\begin{tabular}{cccccc}
\hline 温度 & 时间 & 克隆胚胎 $/$ 个 & 卵裂数 & 4 -细胞数 & 囊胚数 \\
\hline 室温 & $30 \mathrm{~min}$ & 308 & $211\left(68.51^{\mathrm{A}}\right)$ & $126\left(59.72^{\mathrm{A}}\right)$ & $22\left(17.46^{\mathrm{A}}\right)$ \\
$4^{\circ} \mathrm{C}$ & $24 \mathrm{~h}$ & 109 & $79\left(72.48^{\mathrm{B}}\right)$ & $42\left(53.16^{\mathrm{B}}\right)$ & $8\left(19.05^{\mathrm{B}}\right)$ \\
\hline
\end{tabular}

a) 括号内数字表示不同时期克隆胚胎的发育率 $(\%)$, 同列内不同上标间无显著差异, $P>0.05$

\section{3 不同处理的体细胞克隆胚胎移植结果比较}

实验将体外发育的 4-细胞期(图 3)克隆胚胎 102 枚移植到自然发情 $2 \sim 3 \mathrm{~d}$ 的受体山羊输卵

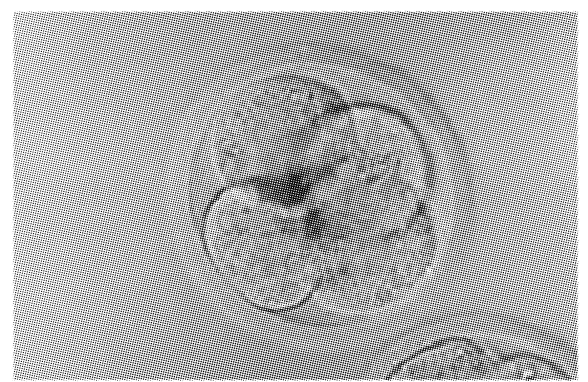

图 3 山羊耳皮肤成纤维细胞克隆胚胎体 外发育的 4-细胞 管内, 结果移植的 84 枚未冷藏体细胞克隆胚胎未能 产仔(表 3), 而移植的 18 枚冷藏体细胞克隆胚胎得 到 1 只发育足月的克隆山羊 $\mathrm{SC} 1(2000$ 年 6 月 16 日)(图 4), 但出生后不久死亡. 解剖结果显示, 肺部 充血造成呼吸困难窒息而死. 将 19 枚囊胚(图 5)移 植到自然发情 6 8 d 的受体山羊子宫角, 结果无论 供体细胞冷藏处理与否都未产仔. 将非冷藏处理的 体细胞克隆山羊桑椹胚移植到自然发情 $5 \mathrm{~d}$ 的受体 山羊子宫角, 结果产羔一只 SC2(2000 年 6 月 22 日)(图 6), 这只克隆山羊健康存活.

表 3 不同日龄体外发育山羊体细胞克隆胚胎的移植比较

\begin{tabular}{|c|c|c|c|c|}
\hline 细胞处理 & 移植胚龄 & 移植胚数 & 受体数 & 产仔数 ${ }^{\mathrm{a}}$ \\
\hline 室温 & 4-细胞 & 84 & 14 & 0 \\
\hline $4^{\circ} \mathrm{C}(24 \mathrm{~h})$ & 4-细胞 & 18 & 3 & $1(5.5)$ \\
\hline 室温 & 囊胚 & 15 & 7 & 0 \\
\hline $4^{\circ} \mathrm{C}(24 \mathrm{~h})$ & 囊胚 & 4 & 2 & 0 \\
\hline 室温 & 桑椹胚 & 18 & 3 & $1(5.5)$ \\
\hline
\end{tabular}

a) 括号内数字表示产仔率 $(\%)$ 




图 4 山羊耳皮肤成纤维细胞克隆胚胎体外 发育的囊胚

\section{4 克隆山羊个体微卫星 DNA 检测分析结果} 实验用 5 对微卫星引物 SR-CRSP1, SR-CRSP5, SR-CRSP6, SR-CRSP7, SR-CRSP24 进行 PCR 扩增, 结果表明, 扩增的 DNA 片段在 3 个对照青山羊不 同个体之间存在一定差异, 而两只克隆山羊与供 体细胞的扩增结果完全相同，但其与受体母羊和 同种对照山羊都存在差异(图 7)(待发表)。这一结 果证实克隆山羊来源于供体体细胞, 同时也表明 这 5 对引物 PCR 扩增的产物在同种不同个体青山 羊之间存在多态性差异.

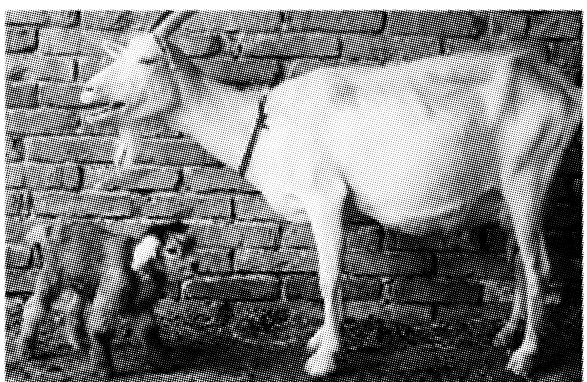

图 $54^{\circ} \mathrm{C}$ 冷藏耳成纤维细胞克隆山羊 $\mathrm{SC} 1$ 和受体母亲

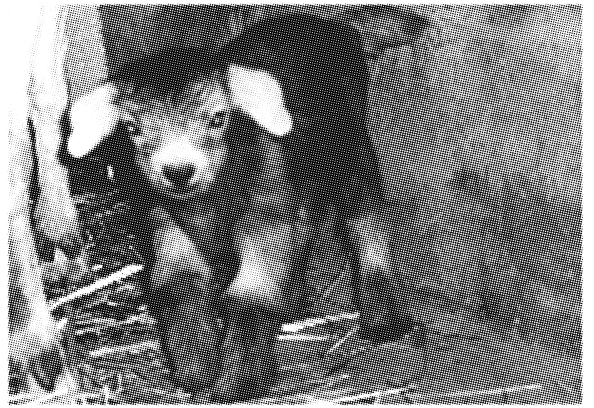

图 6 非冷藏耳成纤维细胞克隆山羊 SC2

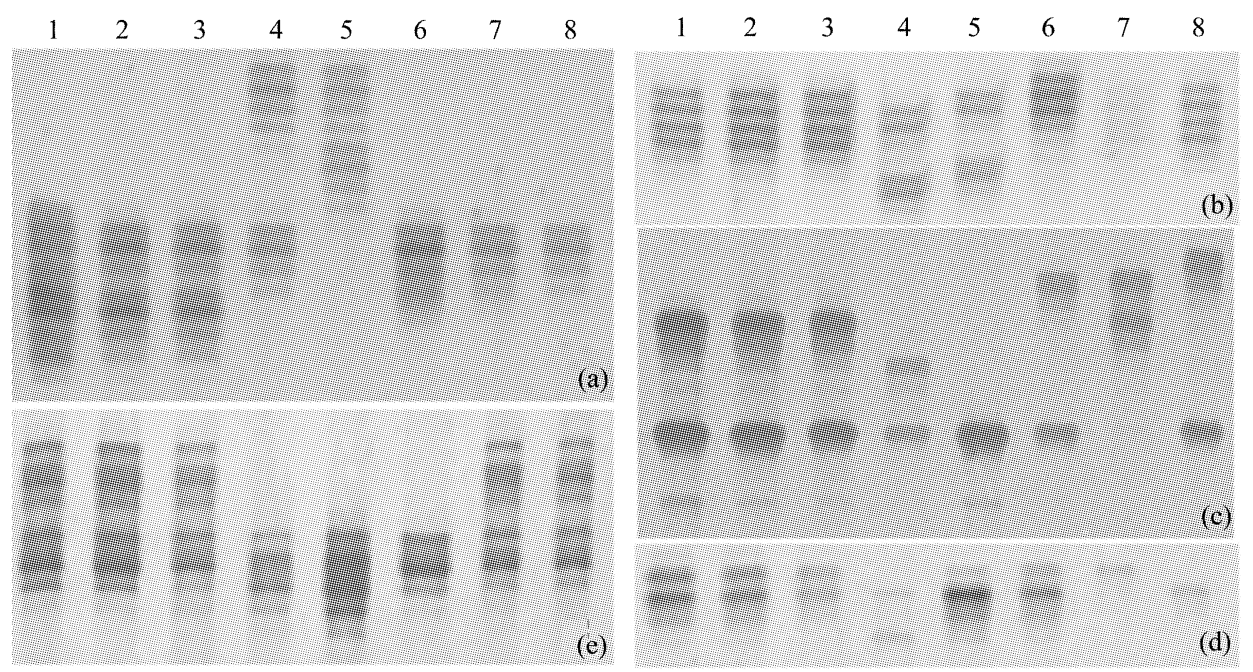

图 7 耳成纤维细胞克隆山羊微卫星 PCR 测序胶电泳

(a) (e)分别为 SR-CRSP-1, SR-CRSP-5, SR-CRSP-6, SR-CRSP-7, SR-CRSP-24 引物的 PCR 产物. 1 8 分别为成 年耳细胞克隆山羊 $\mathrm{SC} 1, \mathrm{SC} 2$, 供体细胞, $\mathrm{SC} 1$ 受体母亲, $\mathrm{SC} 2$ 受体母亲, 对照青山羊母亲、父亲和女儿

\section{3 讨论}

重建肧胎的激活是哺乳动物核移植成功的关键. 通常报道的核移植都是由电诱导完成供 
体细胞的植人, 因此在电融合时就完成了克隆胚胎的激活 ${ }^{[11,14]}$. 电激活效率较低, 而且电激活 常常有赖于卵母细胞成熟后时间 ${ }^{[25]}$. 化学激活的应用使克隆的效率得到显著提高 ${ }^{[26,27]}$. 我们 研究用离子酶素联合 6-DMAP 激活山羊克隆胚胎, 获得了好的激活率, 而且用此激活方法激 活体细胞克隆胚胎也得到了后代, 说明离子酶素联合 6-DMAP 可用于山羊体细胞克隆胚胎的 激活. 本研究所用卵母细胞胞质内直接注射法核移植不同于现在流行的电融合方法, 结果表 明该方法可用于山羊体细胞克隆.

第 1 只成年体细胞克隆绵羊利用的是成年乳腺上皮 ${ }^{[1]}$, 后来的研究结果表明不同成年体细 胞类型对核移植有很大影响. 如小鼠睪丸支持细胞和脑神经细胞不能克隆出活的个体, 而卵 丘细胞可以克隆得到克隆小鼠 ${ }^{[2]}$. 但 Kato 等人 ${ }^{[28]}$ 的研究却没有得到卵丘细胞克隆小鼠, 只得 到了滤泡上皮细胞克隆小鼠, 这也说明不同研究条件下的研究结果也不同. 目前为止, 体细胞 克隆所用供体细胞在核移植前都没有经过低温处理, 我们的研究结果表明, 成年山羊皮肤成 纤维细胞可以用胞质直接注射法克隆得到体细胞克隆山羊, 而且经过低温冷藏后更有利于重 建胚胎的进一步发育. 但我们用培养传代的颗粒细胞克隆却没有得到后代(待发表).

本研究共移植受体奶山羊 29 只, 得到两只后代. 一只来源于非冷藏处理皮肤成纤维细胞 克隆桑椹胚; 另一只来源于 $4^{\circ} \mathrm{C}$ 冷藏 $24 \mathrm{~h}$ 成纤维细胞克隆 4-细胞肧. 虽然统计学分析表明供体 细胞 $4^{\circ} \mathrm{C}$ 冷藏 $24 \mathrm{~h}$ 后克隆的胚胎与非冷藏细胞克隆的胚胎卵裂率和囊胚发育率没有差异, 但 移植结果暗示供体细胞 $4^{\circ} \mathrm{C}$ 冷藏后有利于山羊体细胞克隆, 原因还有待进一步研究. 这一点至 少可以说明体细胞在核移植前 $4^{\circ} \mathrm{C}$ 冷藏是可行的. 本研究获得了冷藏处理供体细胞克隆的哺 乳动物, 推测这样的处理有利于体细胞核在卵母细胞内完成正确重塑.

本研究用了 10 对山羊微卫星引物进行 PCR 扩增分析, 结果有 5 对引物显示出同种不同个 体之间的多态性差异. 研究结果也表明, 用 $6 \%$ 的测序胶和银染完全可以进行微卫星 DNA多态 性分析，也可用于进行个体亲缘关系的分析和克隆动物的鉴定.

致谢 本研究工作在西北农林科技大学完成. 感谢第四军医大学陈苏民教授、陈南春高级 实验师和柴玉波博士在克隆山羊微卫星 DNA 鉴定中给予的大力协助; 感谢王新庄博士、刘泽 隆博士在山羊胚胎移植中提供的帮助.

\section{参考文献}

1 Wilmut I, Schnieke A E, McWhir J, et al. Viable offspring derived from fetal and adult mammalian cells. Nature, 1997, 385 : $810 \sim 813$

2 Wakayama T, Perry A C, Zuccotti M, et al. Full-term development of mice from enucleated oocytes injected with cumulus cell nuclei. Nature, 1998, 394: 369 374

3 Kato Y, Tani T, Sotomaru Y, et al. Eight calves cloned from somatic cells of a single adult. Science, 1998, 282: 2095 2098

4 Wells D N, Misica P M, Tervit H R, et al. Adult somatic cell nuclear transfer is used to preserve the last surviving cow of the enderby Island cattle breed. Reprod Fertil Dev, 1998, 10: 369 378

5 Zakhartchenko V, Alberio R, Stojkovic M, et al. Adult cloning in cattle: potential of nuclei from a permanent cell line and from primary cultures. Mol Reprod Dev, 1999, 54: 264 272

6 Shiga K, Fujita T, Hirose K, et al. Production of calves by transfer of nuclei from cultured somatic cells obtained from Japanese black bulls. Theriogenlogy, 1999, 52: 527 535

7 Schnieke A E, Kind A J, Ritchie W A, et al. Human factor IX transgenic sheep produced by transfer of nuclei from 
transfected fetal fibroblasts. Science, 1997, 278: 2130 2133

8 Cibelli J B, Stice S L, Golueke P J, et al. Cloned transgenic calves produced from nonquiescent fetal fibroblasts. Science, 1998, 280: 1256 1258

9 Zakhartchenko V, Durcova-Hills G, Stojkovic M, et al. Effects of serum starvation and re-cloning on the efficiency of nuclear transfer using bovine fetal fibroblasts. J Reprod Fertil, 1999, 115: 325 331

10 Vignon X, LeBourhis D, Chesne P, et al. Development of bovine nuclear transfer embryos reconstituted with quiescent and proliferative skin fibroblasts. Theriogenology, 1999, 51: 216

11 Baguisi A, Behboodi E, Melican D T, et al. Production of goats by somatic cell nuclear transfer. Nat Biotechnol, 1999, 17: 456 461

12 Onishi A, Iwamoto M, Akita T, et al. Pig cloning by microinjection of fetal fibroblast nuclei. Science, 2000, 289: 1188 1190

13 Kubota C, Yamakuchi H, Todoroki J, et al. Six cloned calves produced from adult fibroblast cells after long-term culture. Proc Natl Acad Sci USA, 2000, 97: 990 995

14 王玉阁, 邹贤刚, 成国祥, 等. 由胎儿成纤维细胞而来的克隆山羊(Capra hircus). 科学通报, 1999, 44(21): 2319 2323

15 Keefer C L, Baldassarre H, Keyston R, et al. Generation of dwarf goat (Capra hircus) clones Following nuclear transfer with transfected and nontransfected fetal fibroblasts and in vitro-matured oocytes. Biol Reprod, 2001, 64: 849 856

16 郭继粀, 李裕强, 张 涌, 从屠宰山羊卵巢制备核移植受体卵母细胞的研究. 西北农业学报, 1998, 7(5): 127 130

17 李裕强, 张 涌, 郭继乍, 等. 山羊皮肤组织细胞分离与培养的研究. 农业生物技术学报, 2000, 8(2): 133 137

18 Darling D C, Morgan S J. Animal Cells: Culture and Media. New York: John Wiley and Sons, Inc, 1994

19 Goto K, Tanaka M, Ookutsu S, et al. Production of a nuclear transferred calf by the intracytoplasmic injection of donor cells. J Reprod Dev, 1997, 43: 257 260

20 Rosenkrans C F, First N L. Culture of bovine zygotes to the blastocyst stage: effects of amino acids and vitamins. Theriogenology, 1991, 35: 266

21 Arevalo E, Holder D A, Derr J N, et al. Caprine microsatellite dinucleotide repeat polymorphisms at the SR-CRSP-1, SR-CRSP-2, SR-CRSP-3, SR-CRSP-4, and SR-CRSP-5 loci. Anim Genet, 1994, 25: 202

22 Bhebhe E, Kogi J, Holder D A, et al. Caprine microsatellite dinucleotide repeat polymorphisms at the SR-CRSP-6, SR-CRSP-7, SR-CRSP-8, SR-CRSP-9, and SR-CRSP-10 loci. Anim Genet, 1994, 25: 203

23 Yeh C C, Kogi J K, Holder M T, et al. Caprine microsatellite dinucleotide repeat polymorphisms at the SR-CRSP21, SR-CRSP22, SR-CRSP23, SR-CRSP24, SR-CRSP25, SR-CRSP26 and SR-CRSP27 loci. Anim Genet, 1997, 28: 380 381

24 李德葆, 周雪平, 许建平, 等. 基因工程操作技术. 上海: 上海科学技术出版社, 1996. 101 111

25 Yang X, Jiang S, Farrell P, et al. Nuclear transfer in cattle: effect of nuclear donor cells, cytoplast age, co-culture, and embryo transfer. Mol Reprod Dev, 1993, 35: 29 36

26 Liu L, Ju J C, Yang X. Parthenogenetic development and protein patterns of newly matured bovine oocytes after chemical activation. Mol Reprod Dev, 1998, 49: 298 307

27 Wells D N, Misica P M, Tervit H R. Production of cloned calves following nuclear transfer with cultured adult mural granulosa cells. Biol Reprod, 1999, 60: 996 1005

28 Kato Y, Yabuuchi A, Motosugi N, et al. Developmental potential of mouse follicular epithelial cells and cumulus cells after nuclear transfer. Biol Reprod, 1999, 61: 1110 1114 